\title{
Low-level viremia and cirrhotic complications in patients with chronic hepatitis $B$ according to adherence to entecavir
}

Seung Bum Lee', Joonho Jeong', Jae Ho Park', Seok Won Jung ${ }^{1}$, In Du Jeong ${ }^{1}$, Sung-Jo Bang ${ }^{1}$, Jung Woo Shin', Bo Ryung Park ${ }^{2}$, Eun Ji Park ${ }^{2}$, and Neung Hwa Park ${ }^{1,2}$

${ }^{1}$ Department of Internal Medicine, ${ }^{2}$ Biomedical Research Center, Ulsan University Hospital, University of Ulsan College of Medicine, Ulsan, Korea

\section{Graphical Abstract}

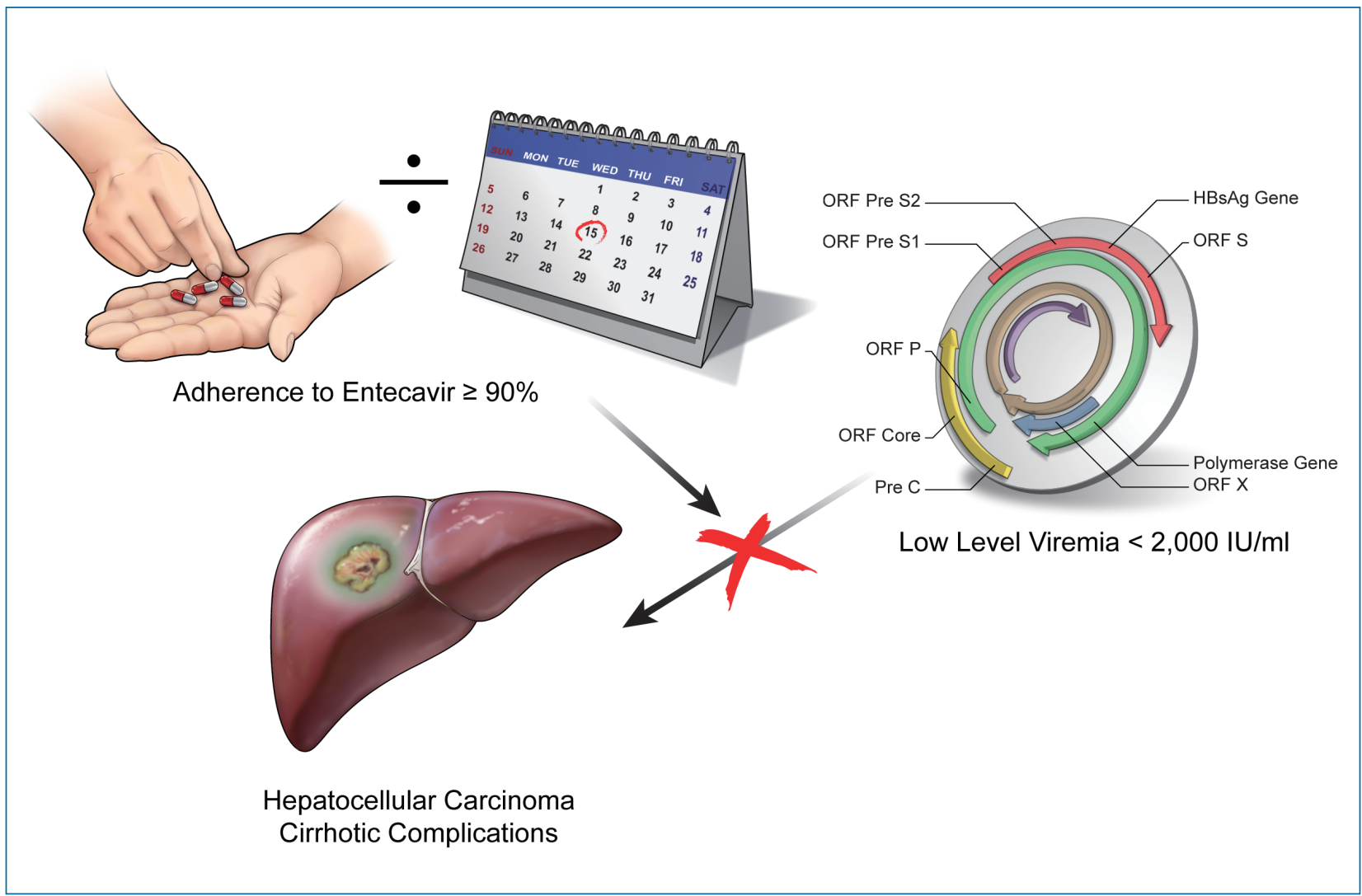

\section{Abbreviations:}

anti-HBe, antibodies to hepatitis B e antigen; $\mathrm{CHB}$, chronic hepatitis $\mathrm{B} ; \mathrm{DM}$, diabetes mellitus; ETV, entecavir; $\mathrm{HBeAg}$, hepatitis $\mathrm{B}$ e antigen; $\mathrm{HBsAg}$, hepatitis $\mathrm{B}$ surface antigen; $H B V$, hepatitis B virus; $H C C$, hepatocellular carcinoma; $H R$, hazard ratio; LLV, low-level viremia; MVR, maintained virologic response; NA, nucleos(t) ide analog; RFMP, restriction fragment mass polymorphism; TDF, tenofovir disoproxil fumarate; $V B T$, virologic breakthrough; $V R$, virologic response

\section{Corresponding author : Neung Hwa Park}

Department of Internal Medicine, Ulsan University Hospital, University of Ulsan College of Medicine, 877 Bangeojinsunhwan-doro, Dong-gu, Ulsan 44033, Korea

Tel: +82-52-250-7029, Fax: +82-52-250-7048

E-mail:nhpark@uuh.ulsan.kr

https://orcid.org/0000-0002-5648-9189 
Background/Aims: Low-level viremia (LLV) after nucleos(t)ide analog treatment was presented as a possible cause of hepatocellular carcinoma (HCC) in patients with chronic hepatitis B (CHB). However, detailed information on patients' adherence in the real world was lacking. This study aimed to evaluate the effects of LLV on HCC development, mortality, and cirrhotic complications among patients according to their adherence to entecavir (ETV) treatment.

Methods: We performed a retrospective observational analysis of data from 894 consecutive adult patients with treatment-naïve CHB undergoing ETV treatment. LLV was defined according to either persistent or intermittent episodes of $<2,000 \mathrm{IU} / \mathrm{mL}$ detectable hepatitis B virus DNA during the follow-up period. Good adherence to medication was defined as a cumulative adherence $\geq 90 \%$ per study period.

Results: Without considering adherence in the entire cohort $(n=894)$, multivariate analysis of the HCC incidence showed that LLV was an independent prognostic factor in addition to other traditional risk factors in the entire cohort $(P=0.031)$. Good adherence group comprised 617 patients (69.0\%). No significant difference was found between maintained virologic response and LLV groups in terms of the incidence of liver-related death or transplantation, HCC, and hepatic decompensation in good adherence group, according to multivariate analyses.

Conclusions: In patients with treatment-naïve CHB and good adherence to ETV treatment in the real world, LLV during treatment is not a predictive factor for HCC and cirrhotic complications. It may be unnecessary to adjust their antiviral agent for patients with good adherence who experience LLV during ETV treatment. (Clin Mol Hepatol 2020;26:364375)

Keywords: Hepatitis B; Medication adherence; Carcinoma, Hepatocellular; Liver cirrhosis

\footnotetext{
Study Highlights

- Nonadherence to medication and low-level viremia (LLV) can restrict the full benefits of antiviral treatment for chronic hepatitis B.

- In patients with good adherence ( $\geq 90 \%)$ to entecavir treatment in the real-world, LLV during treatment was not a risk factor for hepatocellular carcinoma and cirrhotic complications.

- It is helpful to check the adherence of the patient with a suboptimal virological response, prior to considering adjusting their antiviral agent immediately and for patients with good adherence, it may be unnecessary and careful observation will be possible.
}

\section{INTRODUCTION}

Effective antiviral nucleos(t)ide analog (NA) treatment for chronic hepatitis B (CHB) using potent drugs with a high genetic barrier, such as entecavir (ETV) or tenofovir disoproxil fumarate (TDF), has been shown to regress hepatic fibrosis, prevent liverrelated complications, and improve patient survival. ${ }^{1-6}$ However, the risk of hepatic complications, particularly the development of hepatocellular carcinoma (HCC), in CHB was not fully eliminated, even with potent agents. ${ }^{7-11}$ Low-level viremia (LLV) has been suggested as a possible cause of HCC in patients receiving NA treatment. ${ }^{12,13}$ According to Kim et al., ${ }^{13}$ LLV, defined as a lack of maintained virologic response (MVR) during ETV monotherapy, was associated with a higher risk of HCC, especially in those with cirrhosis. However, the recently updated American Association for the Study of the Liver Disease guidelines recommend that patients with LLV who are on ETV or TDF monotherapy continue monotherapy. ${ }^{14}$ Furthermore, nonadherence to NA treatment may lead to treatment failure. Adherence to treatment refers to the extent to which a patient takes medication as prescribed and for the duration of treatment agreed upon by the patient and physician. ${ }^{15,16}$ In real-world clinical settings, nonadherence is common and can restrict the full benefits of antiviral treatment. ${ }^{17-19}$ In a previous study, poor treatment adherence was demonstrated to be a significant risk factor for mortality, HCC, and hepatic decompensation. ${ }^{20}$ Treatment nonadherence is likely to be a more significant contributor to treatment failure than antiviral resistance against anti-hepatitis B virus (HBV) agents, such as ETV, which exhibit potent viral suppression with a lower risk of drug resistance. ${ }^{18,19}$ This study aimed to evaluate the effect of LLV on HCC development, mortality, and cirrhotic complications in patients with CHB according to their adherence to ETV medication in real-world clinical settings. 


\section{PATIENTS AND METHODS}

\section{Patients}

In this retrospective longitudinal observational study, we used the electronic medical records of patients with treatment-naïve CHB who received ETV therapy between January 1, 2007 and January 31, 2017 at Ulsan University Hospital, a tertiary referral center in South Korea. Patients were eligible for inclusion at the time of the initiation of antiviral treatment if they met the following criteria: age $\geq 18$ years; hepatitis $B$ surface antigen ( $\mathrm{HBs} A g$ )positive for $>6$ months or CHB defined by clinical history; no malignancy including HCC at baseline; no evidence of viral coinfections (i.e., human immunodeficiency virus, hepatitis $\mathrm{C}$ virus, or hepatitis D virus); no alcohol-related liver disease or autoimmune hepatitis; and treatment-naïve prior to starting ETV $0.5 \mathrm{mg}$ per day (ETV has been available in South Korea since January 1 , 2007). The exclusion criteria included the following: follow-up duration <1 year; evidence of decompensated cirrhosis as indicated by the presence (or history) of ascites, esophageal, or gastric variceal bleeding, hepatic encephalopathy, spontaneous bacterial peritonitis, hepatorenal syndrome, liver transplantation, or a Child-Pugh score $\geq 7$; serum creatinine level $>1.5 \mathrm{mg} / \mathrm{dL}$ at baseline; HBV DNA <2,000 IU/L at baseline; or death within 6 months of treatment initiation. Patients who developed HCC within the first year of enrollment were also excluded to minimize the inclusion of pre-existing unidentified HCC and the misattribution of treatment effect. We also excluded patients with renal disease who needed dose adjustments that could affect the adherence rate. Finally, 894 patients were enrolled. Information about baseline patient characteristics and clinical outcomes were obtained from complete inpatient and outpatient medical records. This cohort was originally investigated by Shin et al. ${ }^{20}$ and clinical progress was further updated through August 31, 2018 for the current study. This study was approved by the Institutional Review Board of Ulsan University Hospital (\#IRB No. 06-2017-26). The requirement for informed consent was waived, as patient records and information were de-identified prior to analysis.

\section{Serum assay}

Whole blood count, biochemical and HBV virologic markers, and serum HBV DNA levels were assessed for all patients every 3-6 months during ETV therapy. Serum HBV DNA levels were quantified using the $\operatorname{COBAS}^{\circledR}$ TaqMan HBV test (Roche, Branch- burg, NJ, USA), which has a lower detection limit of $12 \mathrm{IU} / \mathrm{mL}$ (60 copies/mL). Levels of HBsAg, hepatitis B e-antigen (HBeAg), and antibodies to hepatitis B e-antigen (anti-HBe) were examined using an enzyme immunoassay. The virologic outcomes included virologic response (VR), virologic breakthrough (VBT), and MVR or LLV during the on-treatment follow-up period. VR was defined as undetectable serum HBV DNA by polymerase chain reaction assay ( $<12 \mathrm{IU} / \mathrm{mL}$ ) for two consecutive measurements during ETV treatment. VBT was defined as an increase of $>1 \log _{10} \mathrm{IU} / \mathrm{mL}$ in serum HBV DNA level from nadir for two consecutive measurements or on the last available measurement. Based on the serum HBV DNA levels during the follow-up period, patients were categorized as either MVR or LLV at the last follow-up, as mentioned in a previous study by Kim et al..$^{13}$ MVR was defined as having serum HBV DNA persistently undetectable throughout the follow-up period after achieving a VR. The remaining patients showed either persistent or intermittent episodes of detectable serum HBV DNA $<2,000 \mathrm{IU} / \mathrm{mL}$ during follow-up, which was defined as LLV. Restriction fragment mass polymorphism (RFMP; Genematrix ${ }^{\circledR}$, Youngin, Korea) was used to identify ETV-resistant mutations in the HBV polymerase gene following VBT occurrence during the treatment period. The RFMP assay can detect 100 copies of HBV genome/mL. ${ }^{21}$ For this study, we have further investigated and updated the history of diabetes mellitus (DM) and hypertension. DM was defined as a self-reported history of diabetes, use of antidiabetic medication, and/or those with a fasting plasma glucose $\geq 126 \mathrm{mg} / \mathrm{dL}$. Hypertension was defined as blood pressure $\geq 140 / 90 \mathrm{mmHg}$ or a self-reported history of hypertension and/or use of anti-hypertensive medication.

\section{Follow-up assessments}

During the follow-up period, all patients underwent periodic surveillance with ultrasonography and laboratory workups, including a-fetoprotein and protein-induced vitamin $\mathrm{K}$ absence or antagonist-II, every 6 months to screen for HCC. Liver cirrhosis was clinically defined based on repeated liver imaging studies (nodular liver surface or caudate lobe hypertrophy) with thrombocytopenia $\left(<150 \times 1,000 / \mathrm{mm}^{3}\right)$ or splenomegaly (by imaging), and/or by the presence of varices (by upper endoscopy or imaging studies). ${ }^{22}$ HCC diagnosis was confirmed using radiology (dynamic computed tomography and/or magnetic resonance imaging), as recommended by international guidelines. ${ }^{23}$ The clinical outcomes were the cumulative incidence of liver-related death or transplantation, HCC, and hepatic decompensation (ascites, variceal bleeding, 
spontaneous bacterial peritonitis, hepatic encephalopathy, and hepatorenal syndrome). Liver-related death was defined as death related to cirrhotic complications and HCC. To avoid statistical repetition, the earliest cirrhotic complication was selected if a given patient experienced different types of cirrhotic complications at different time points.

\section{Medication adherence}

Medical and pharmacy refill records were reviewed to assess medication adherence by prescription records and the actual amount of medication taken by the patient. Adherence rate was expressed as the percentage of days the patient had medications in his/her possession during the period in which he/she was undergoing therapy. This proportion was calculated as the sum of days on which medication was supplied (obtained over a series of intervals) divided by the total treatment duration (days), which was derived from the dates of the first and last prescriptions dispensed. The medication from the final prescription refill was not included, as its consumption was unknown. Good adherence to medication was defined as a cumulative adherence $\geq 90 \%$ per study period among patients who were prescribed ETV in a given period. The cutoff of $90 \%$ was previously used in a similar study. ${ }^{20,24}$

\section{Statistical analyses}

Continuous variables were compared using the Student's $t$-test or Mann-Whitney U-test, and categorical variables were compared using the chi-square test or Fisher's exact test. Serum HBV DNA (IU/mL) levels were logarithmically transformed for analyses. All statistical tests were considered significant with two-sided $P$-values of $<0.05$. Cumulative probabilities of the clinical outcomes were calculated using the Kaplan-Meier method. Univariate and multivariate analyses for factors predictive of the development of HCC were performed using a Cox proportional-hazard model. The results of the model were presented as a hazard ratio $(\mathrm{HR})$ with a 95\% confidence interval $(\mathrm{Cl})$. Variables were not included in the multivariate model if $P$-values in the univariate analysis were $>0.2$. Statistical analyses were performed using the statistical package SPSS for Windows (version 24.0; SPSS Inc., Chicago, IL, USA).

\section{RESULTS}

\section{Patient characteristics and virologic outcomes in the entire cohort}

Overall, 1,955 patients with NA-naïve CHB were consecutively treated with ETV during the study period from January 1, 2007 to August 31, 2018. Among these patients, 894 were considered eligible for analysis (Supplementary Fig. 1). The demographic and clinical characteristics of all patients included in this study are summarized in Table 1. VR was observed in 812 patients $(90.8 \%)$. The median time to VR was 0.8 years (range, $0.1-6.6$ ). The cumu-

Table 1. Baseline characteristics in all patients

\begin{tabular}{|c|c|c|c|c|}
\hline Characteristic & Overall $(n=894)$ & $\operatorname{MVR}(n=654)$ & $\operatorname{LLV}(n=240)$ & $P$-value \\
\hline Age (years) & $52.0 \pm 11.4$ & $52.4 \pm 11.2$ & $50.8 \pm 11.7$ & 0.068 \\
\hline Male sex & $597(66.8)$ & 418 (63.9) & $179(75.6)$ & 0.003 \\
\hline HBeAg positivity & $537(60.1)$ & $362(55.4)$ & $175(72.9)$ & $<0.001$ \\
\hline Cirrhosis & $440(49.2)$ & $330(50.5)$ & $110(45.8)$ & 0.220 \\
\hline HBV DNA $\left(\log _{10} \mid \mathrm{U} / \mathrm{mL}\right)$ & $6.4 \pm 1.4$ & $6.3 \pm 1.4$ & $6.6 \pm 1.5$ & 0.011 \\
\hline ALT (IU/mL) & $143.7 \pm 173.1$ & $143.6 \pm 177.6$ & $141.9 \pm 158.5$ & 0.890 \\
\hline Albumin (g/dL) & $4.2 \pm 0.4$ & $4.2 \pm 0.4$ & $4.1 \pm 0.5$ & 0.093 \\
\hline Prothrombin time (INR) & $1.0 \pm 0.2$ & $1.0 \pm 0.2$ & $1.0 \pm 0.3$ & 0.377 \\
\hline Platelet $\left(\times 1,000 / \mathrm{mm}^{3}\right)$ & $169.8 \pm 63.1$ & $169.9 \pm 63.0$ & $169.1 \pm 63.0$ & 0.872 \\
\hline Diabetes mellitus & $150(16.8)$ & $105(16.1)$ & $45(18.8)$ & 0.339 \\
\hline Hypertension & $98(11.0)$ & $74(11.3)$ & $24(10.0)$ & 0.630 \\
\hline
\end{tabular}

Values are presented as mean \pm standard deviation or number (\%).

MVR, maintained viral response; LLV, low-level viremia; HBeAg, hepatitis B e-antigen; HBV, hepatitis B virus; ALT, alanine aminotransferase; INR, international normalized ratio. 
lative VR incidence rates were $66.3 \%, 85.2 \%$, and $95.2 \%$ at 1,3 , and 5 years, respectively. Among the patients, MVR was achieved in 654 (73.2\%), and LLV remained in 240 (26.8\%). During followup, 125 patients (14.0\%) experienced VBT (good adherence group, $n=30$; poor adherence group, $n=95)$, with lower rates in good adherence group than in poor adherence group $(4.9 \%$ vs. $34.3 \%, P<0.001)$. The cumulative probability of VBT was $0.6 \%$, $6.7 \%, 10.8 \%$, and $12.4 \%$ at years $1,3,5$, and 7 , respectively. Good adherence to ETV medication was observed in 617 patients (69.0\%), and 277 patients (31.0\%) had poor adherence. Among the patients with good adherence, MVR was achieved in 509 $(82.5 \%)$, and LLV remained in 108 (17.5\%) (Table 2). Kaplan-Meier survival analysis demonstrated a significantly higher risk of LLV in poor adherence group than in good adherence group (log-rank $P<0.001$; Fig. 1). There were a total of 42 cases of HBV DNA $\geq 2,000 \mathrm{IU} / \mathrm{mL}$ in $125 \mathrm{VBT}$ patients. Among them, 17 patients (including 11 patients with ETV-resistant mutations) were eventually replaced by TDF, at which point the follow-up for the study was censored. For the remaining 25 patients, at HBV DNA $\geq 2,000$ $\mathrm{IU} / \mathrm{mL}$, the compliance was investigated to encourage ETV medication. As a result, within 3 months (next follow-up test) for 23 and within 6 months for two patients, HBV DNA was reduced to below 2,000 IU/mL, in which case ETV treatment was continued. The 25 patients who continued to take ETV even though HBV DNA was $\geq 2,000 \mathrm{IU} / \mathrm{mL}$ did not show $\geq 2,000 \mathrm{IU} / \mathrm{mL}$ again in their subsequent observations, and all of them were considered LLV in this analysis. Among the patients with LLV, 80 underwent muta- tion tests and 22 developed ETV-resistant mutations (L180M/ M204V/S202G [n=13]; L180M/M204V/T184LSM [n=5]; L180M/ M204V/M250L [n=4]). The cumulative probability of acquiring a genotypic ETV mutation was $0 \%, 1.5 \%, 2.7 \%$, and $3.5 \%$ at years $1,3,5$, and 7, respectively. Regarding LLV, 56/240 patients received TDF, and for statistical analysis, those patients were censored after they switched from ETV to TDF. Of the 56 TDF replacement patients, 45 were caused by VBT and 11 were non-VR cases (ETV-resistant mutations were existed in all non-VR cases).

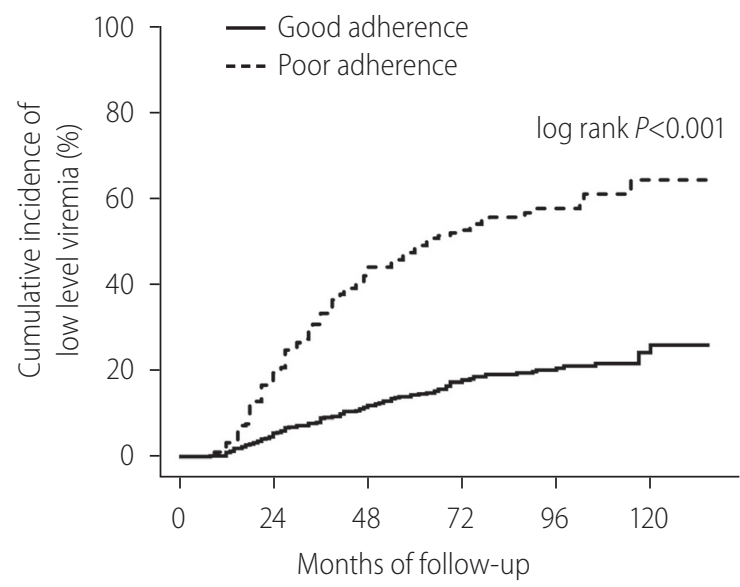

Number at risk $\begin{array}{lllllll}\text { Good adherence } & 617 & 535 & 436 & 332 & 176 & 39\end{array}$ $\begin{array}{lllllll}\text { Pooradherence } & 277 & 199 & 108 & 68 & 35 & 5\end{array}$

Figure 1. Cumulative incidence of low-level viremia according to good adherence vs. poor adherence.

Table 2. Baseline characteristics in good adherence group

\begin{tabular}{|c|c|c|c|c|}
\hline Characteristic & Overall $(n=617)$ & $\operatorname{MVR}(n=509)$ & $\operatorname{LLV}(n=108)$ & $P$-value \\
\hline Age (years) & $52.6 \pm 11.1$ & $52.9 \pm 11.0$ & $51.1 \pm 11.7$ & 0.156 \\
\hline Male sex & $400(64.8)$ & $313(61.5)$ & $87(80.6)$ & $<0.001$ \\
\hline HBeAg positivity & $362(58.7)$ & $281(55.2)$ & $81(75.0)$ & $<0.001$ \\
\hline Cirrhosis & $297(48.1)$ & $257(50.5)$ & $40(37.0)$ & 0.011 \\
\hline HBV DNA $\left(\log _{10} \mid \mathrm{U} / \mathrm{mL}\right)$ & $6.4 \pm 1.4$ & $6.3 \pm 1.4$ & $6.5 \pm 1.5$ & 0.175 \\
\hline $\mathrm{ALT}(\mathrm{IU} / \mathrm{mL})$ & $138.3 \pm 171.8$ & $141.6 \pm 176.8$ & $122.6 \pm 145.2$ & 0.238 \\
\hline Albumin (g/dL) & $4.2 \pm 0.4$ & $4.2 \pm 0.4$ & $4.1 \pm 0.5$ & 0.246 \\
\hline Prothrombin time (INR) & $1.0 \pm 0.2$ & $1.0 \pm 0.2$ & $1.0 \pm 0.1$ & 0.624 \\
\hline Platelet $\left(\times 1,000 / \mathrm{mm}^{3}\right)$ & $171.5 \pm 62.9$ & $170.2 \pm 62.2$ & $177.6 \pm 65.6$ & 0.288 \\
\hline Diabetes mellitus & $100(16.2)$ & $77(15.1)$ & $23(21.3)$ & 0.116 \\
\hline Hypertension & $64(10.4)$ & $55(10.8)$ & $9(8.3)$ & 0.602 \\
\hline
\end{tabular}

Values are presented as mean \pm standard deviation or number (\%).

MVR, maintained viral response; LLV, low-level viremia; HBeAg, hepatitis B e-antigen; HBV, hepatitis B virus; ALT, alanine aminotransferase; INR, international normalized ratio. 


\section{Clinical outcomes of the entire cohort}

The clinical outcomes of the entire cohort, according to MVR vs. LLV, are shown in Figure 2. During the follow-up period of up to 132 months, HCC developed in 91/894 patients (10.2\%): 58/654 $(8.7 \%)$ in MVR group and 33/240 (13.8\%) in LLV group. The Cumulative incidence of HCC was significantly lower in MVR group than in LLV group: 5- and 10-year cumulative incidence rates of HCC in MVR vs. LLV were 7.8 vs. $11.5 \%$ and 12.8 vs. $20.1 \%$, respectively (log-rank $P=0.017$ ). Liver-related death or transplantation occurred in 22/894 patients (2.5\%) (14/654 [2.1\%] in MVR group and $8 / 240$ [3.3\%] in LLV group), and hepatic decompensa-

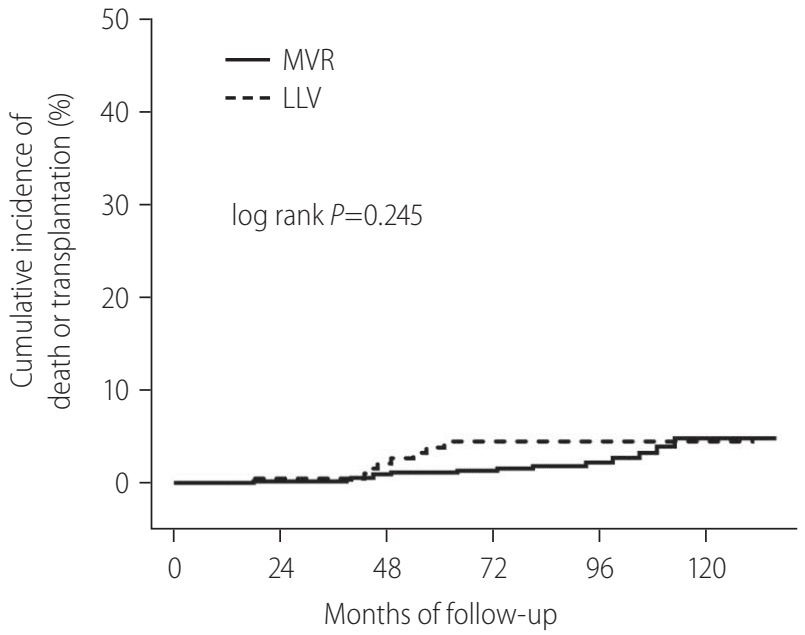

Number at risk

\begin{tabular}{|c|c|c|c|c|c|}
\hline MVR & 654 & 597 & 517 & 420 & 229 \\
\hline LLV & 240 & 210 & 173 & 139 & 80 \\
\hline
\end{tabular}

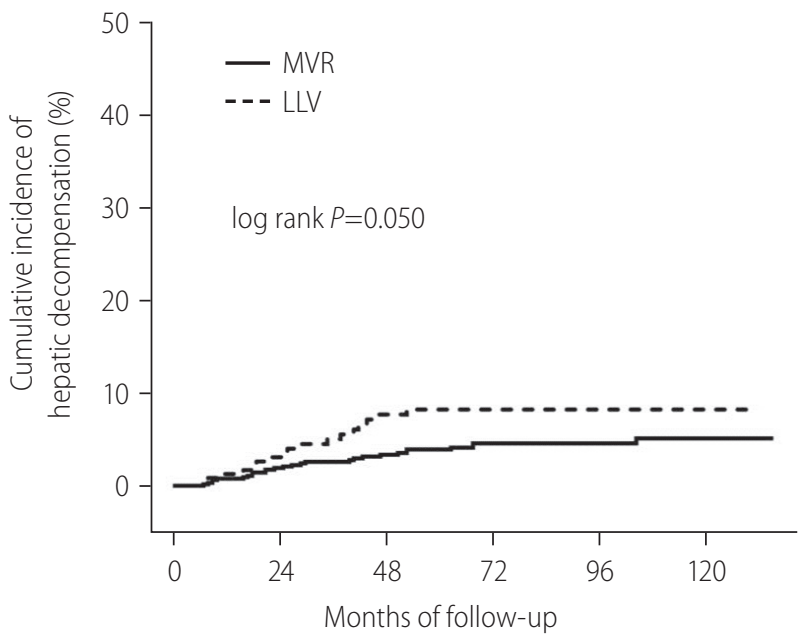

Number at risk

\begin{tabular}{|c|c|c|c|c|c|c|}
\hline MVR & 654 & 586 & 503 & 403 & 219 & 49 \\
\hline LLV & 240 & 205 & 165 & 136 & 78 & 11 \\
\hline
\end{tabular}

tion occurred in 44/894 patients (4.9\%) (27/654 [4.1\%] in MVR group and 17/240 [7.1\%] in LLV group). However, in terms of other clinical outcomes, no statistically significant difference was found between MVR and LLV groups (log-rank $P=0.245$ for liver-related death or transplantation; log-rank $P=0.050$ for hepatic decompensation).

\section{Clinical outcomes of good adherence group}

Clinical outcomes according to MVR vs. LLV in good adherence group are shown in Figure 3. Overall, during the follow-up period of up to 132 months, HCC developed in 43/617 patients (7.0\%):

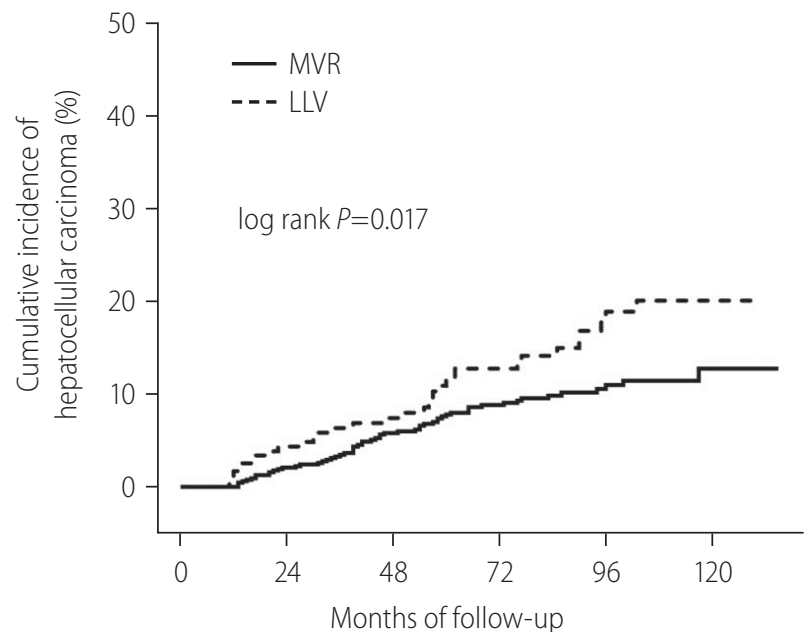

Number at risk

\begin{tabular}{|c|c|c|c|c|c|}
\hline MVR & 654 & 586 & 496 & 390 & 210 \\
\hline LLV & 240 & 201 & 164 & 133 & 75 \\
\hline
\end{tabular}

Figure 2. Cumulative incidence of liver-related death or transplantation $\mathrm{HCC}$, and hepatic decompensation in the entire cohort ( $n=894$ ). (A) Cumulative incidence of liver-related death or transplantation according to MVR vs. LLV. (B) Cumulative incidence of HCC according to MVR vs. LLV. (C) Cumulative incidence of hepatic decompensation according to MVR vs. LLV. MVR, maintained virologic response; LLV, low-level viremia; HCC, hepatocellular carcinoma. 


\section{CLINICAL and MOLECULAR \\ HEPATOLOGY}

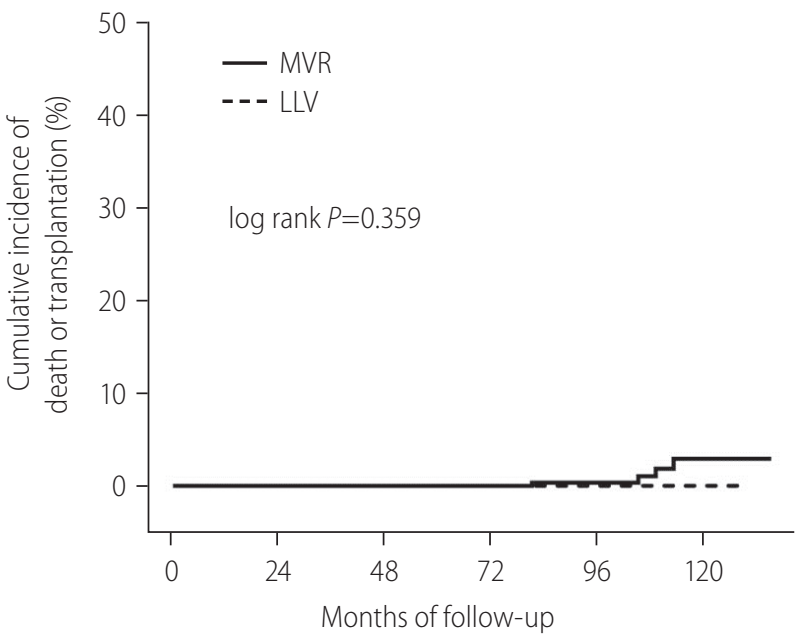

Number at risk

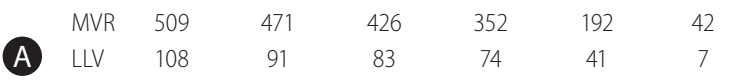

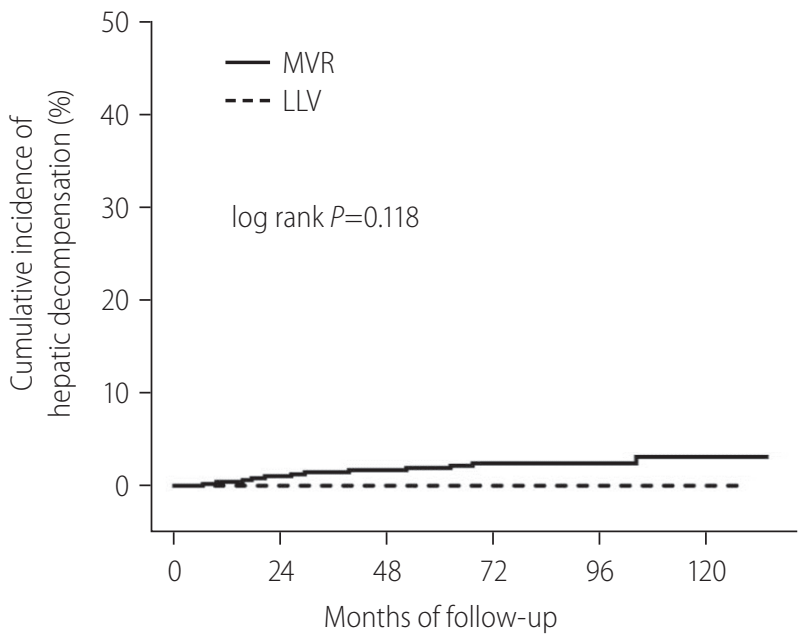

Number at risk

\begin{tabular}{|c|c|c|c|c|c|c|}
\hline MVR & 509 & 466 & 418 & 341 & 185 & 41 \\
\hline LLV & 108 & 91 & 83 & 74 & 41 & 7 \\
\hline
\end{tabular}

36/509 (7.1\%) in MVR group and 7/108 (6.5\%) in LLV group. Liver-related death or transplantation occurred in $4 / 617$ patients (0.6\%) (4/509 [7.9\%] in MVR group and 0/108 [0.0\%] in LLV group), and hepatic decompensation occurred in 12/617 (1.9\%) patients (12/509 [2.4\%] in MVR group and 0/108 [0.0\%] in LLV group). The cumulative incidence of liver-related death or transplantation, HCC, and hepatic decompensation showed no significant differences between MVR and LLV groups (log-rank $P=0.359$ for liver-related death or transplantation; log-rank $P=0.937$ for HCC; log-rank $P=0.118$ for hepatic decompensation). The development of HCC also showed no difference between MVR and LLV groups in the subgroup analysis regarding cirrhotic subcohort

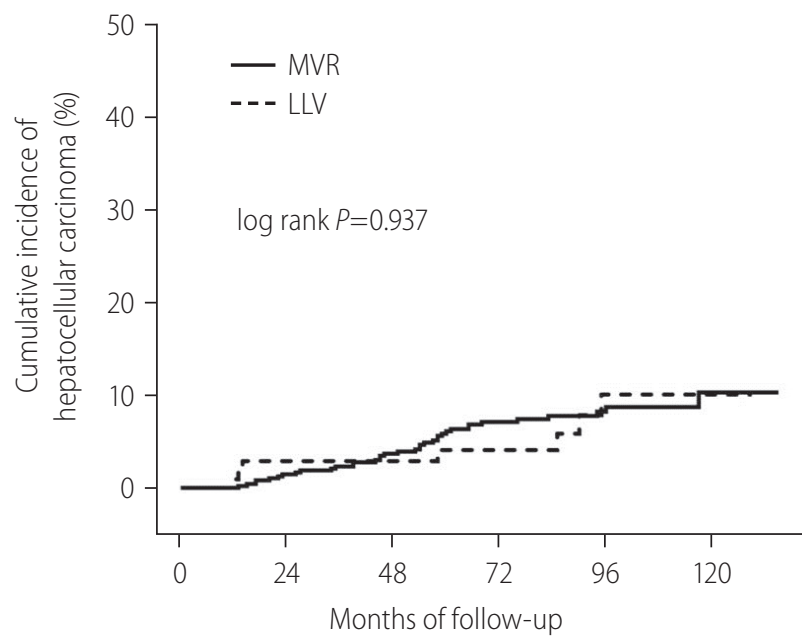

Number at risk

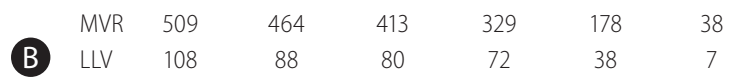

Figure 3. Cumulative incidence of liver-related death or transplantation, HCC, and hepatic decompensation in good adherence group $(n=617)$ (A) Cumulative incidence of liver-related death or transplantation according to MVR vs. LLV. (B) Cumulative incidence of HCC according to MVR vs, LLV. (C) Cumulative incidence of hepatic decompensation according to MVR vs. LLV. MVR, maintained virologic response; LLV, low-level viremia; HCC, hepatocellular carcinoma.

(log-rank $P=0.536)$ (Supplementary Fig. 2).

\section{Risk factors for HCC}

Multivariate analyses were performed to compare the effect of LLV to other well-known risk factors for the development of HCC in the entire cohort and good adherence group (Table 3). Multivariate analysis showed that among the entire cohort, in addition to the traditional risk factors for HCC such as old age, male sex, and cirrhosis, MVR presented a statistically significant association with the development of HCC with a HR of $0.61(95 \% \mathrm{Cl}, 0.39$ 0.96 ; $P=0.031)$. However, in the analyses of good adherence 
Table 3. Multivariate analysis of potential risk factors for hepatocellular carcinoma in the entire cohort and good adherence subcohort

\begin{tabular}{|c|c|c|c|c|}
\hline \multirow{2}{*}{ Characteristic } & \multicolumn{2}{|c|}{ Entire cohort $(n=894)$} & \multicolumn{2}{|c|}{ Good adherence subcohort $(n=617)$} \\
\hline & $\mathrm{HR}(95 \% \mathrm{CI})$ & $P$-value & $\mathrm{HR}(95 \% \mathrm{Cl})$ & $P$-value \\
\hline Age & $1.06(1.03-1.09)$ & $<0.001$ & $1.05(1.01-1.10)$ & 0.024 \\
\hline Male sex & $4.77(2.57-8.87)$ & $<0.001$ & $9.00(3.05-26.60)$ & $<0.001$ \\
\hline HBeAg positivity & $0.86(0.53-1.40)$ & 0.536 & $0.84(0.41-1.71)$ & 0.624 \\
\hline Cirrhosis & $6.10(2.79-13.32)$ & $<0.001$ & $5.18(1.82-14.75)$ & 0.002 \\
\hline MVR & $0.61(0.39-0.96)$ & 0.031 & $1.02(0.44-2.38)$ & 0.967 \\
\hline HBV DNA $\left(\log _{10} 1 \mathrm{U} / \mathrm{mL}\right)$ & $0.91(0.76-1.09)$ & 0.312 & $0.83(0.63-1.09)$ & 0.185 \\
\hline $\mathrm{ALT}(\mathrm{IU} / \mathrm{mL})$ & $1.00(1.00-1.00)$ & 0.184 & $1.00(1.00-1.00)$ & 0.596 \\
\hline Albumin ( $\mathrm{g} / \mathrm{dL})$ & $0.65(0.39-1.08)$ & 0.093 & $0.46(0.19-1.10)$ & 0.079 \\
\hline Prothrombin time (INR) & $1.86(0.72-4.80)$ & 0.200 & $0.38(0.02-6.21)$ & 0.495 \\
\hline Platelet $\left(\times 1,000 / \mathrm{mm}^{3}\right)$ & $1.00(0.99-1.00)$ & 0.613 & $1.00(0.99-1.01)$ & 0.767 \\
\hline Diabetes mellitus & $1.37(0.86-2.20)$ & 0.190 & $1.73(0.87-3.42)$ & 0.118 \\
\hline Hypertension & $2.09(1.27-3.45)$ & 0.004 & $2.82(1.32-6.02)$ & 0.007 \\
\hline
\end{tabular}

$\mathrm{HR}$, hazard ratio; $\mathrm{Cl}$, confidence interval; HBeAg, hepatitis B e antigen; MVR, maintained viral response; HBV, hepatitis B virus; ALT, alanine aminotransferase; INR, international normalized ratio.

group, LLV showed no independent association with HCC $(P=0.967)$, whereas the traditional risk factors for HCC, such as age, male sex, and cirrhosis, presented strong associations with the development of HCC in multivariate analysis.

\section{DISCUSSION}

According to multivariate analysis, LLV during treatment was not a predictive factor for HCC and cirrhotic complications in patients with CHB and good adherence to ETV treatment. With the development of antiviral agents, a dramatic change has occurred in the treatment and prognosis of CHB. In particular, potent antiviral agents with high genetic barriers enabled continued use of medication and showed long-term results in reduced mortality and the progression of cirrhosis or HCC. ${ }^{1-6}$ However, even with the prolonged use of antiviral agents, complications of $\mathrm{CHB}$, particularly HCC, are not fully inhibited. ${ }^{7-11}$ A multicenter study which investigated the incidence of HCC in 1,666 Caucasian patients with CHB receiving ETV or TDF showed that the cumulative probability of $\mathrm{HCC}$ was $3.4 \%$ at year 3 and $8.7 \%$ at year 5 , with an annual incidence rate of $1.37 \%$ per year. ${ }^{25}$ The annual incidence of HCC in Caucasian patients with compensated HBV-related cirrhosis was about 2.2\%, and the 5-year cumulative incidence was about $10 \%{ }^{26}$ Previous studies of Korean patients showed that even with optimal VR under NA therapy, the annual incidence of HCC was
$0.15-0.80 \%$ per year in non-cirrhotic patients, and $0.95-3.55 \%$ per year in compensated-cirrhotic patients. ${ }^{10,27,28}$

LLV has been suggested as a possible cause of HCC in patients receiving NA treatment. ${ }^{12,13}$ A previous study showed that LLV, defined as a lack of MVR, significantly increases the incidence of HCC in patients with CHB who are receiving ETV. ${ }^{13}$ Sub-optimally suppressed viral replication in the liver can cause continuous inflammation, and the proliferation of inserted HBV oncogenes is also considered an important mechanism of liver cancer. ${ }^{29-31}$ In a previous study, patients with LLV in whom a continuous viral response was not achieved had an increased cumulative incidence of HCC, which was more noticeable in patients with cirrhosis. ${ }^{13}$ This was also evident in our study; subgroup analysis of the cirrhotic subcohort showed that the cumulative incidence of $\mathrm{HCC}$ and hepatic decompensation were significantly lower in MVR group than in LLV group (log-rank $P=0.001$ and log-rank $P=0.011$, respectively) (Supplementary Fig. 3).

Meanwhile, in another study, we presented poor treatment adherence as a significant risk factor for mortality, HCC, and hepatic decompensation. ${ }^{20}$ Data on adherence to NA treatment for CHB are limited. In a previous study, a pharmacy claims database with 11,100 patients receiving NAs for CHB was used to investigate the rates of patient adherence in a real-world setting. ${ }^{32}$ The overall adherence rates were found to be $87.8 \%$, with $55.3 \%$ of patients having $\geq 90 \%$ adherence. Other studies have reported mean NA treatment adherence rates ranging from $81 \%$ to $99 \% .{ }^{33,34}$ This 
was consistent with the mean adherence rate of $89.1 \%$ observed in the current study. ${ }^{20}$ Despite the significance of viral suppression to prevent cirrhotic complications and mortality, evidence on the association between adherence to ETV treatment and hepatic complications still remain limited. In our previous study, we evaluated the association of adherence to ETV treatment on hepatic complications in patients with $\mathrm{CHB} .{ }^{20}$ Multivariate Cox proportional regression analyses demonstrated that adherence to ETV treatment was a powerful factor for HCC development, cirrhotic complications, and liver-related or all-cause mortality. In the same study, continuous viral response and MVR increased, whereas VBT was significantly low in patients with good adherence, indicating the correlation between adherence and viral response. These results suggest that poor adherence to antiviral agents is closely linked to LLV. In our current study, when we assessed the correlation between LLV and adherence, LLV was significantly lower in patients with good adherence (Fig. 2). Accordingly, we intended to evaluate the impact of LLV on the risk of HCC, rate of hepatic decompensation, and survival rate in this study in good adherence group and the entire cohort.

According to Kim et al., ${ }^{13}$ MVR was achieved in 498/875 patients (56.9\%) in the study population, and HCC developed in $85 / 875$ patients (9.7\%) within 60 months; these figures possibly included a significant number of patients with poor adherence. Without considering adherence in our entire cohort $(n=894)$, MVR was achieved in 654/894 patients (73.2\%), and HCC developed in $91 / 894$ patients $(10.2 \%)$. This finding was similar to that in a previous report which assessed MVR in 1,466 ETV-treated patients and reported an MVR rate of $78 \%$ in patients with cirrhosis and $77 \%$ in patients without cirrhosis. ${ }^{35}$ This study evaluated LLV as a risk factor for mortality, $\mathrm{HCC}$, and hepatic decompensation in patients with CHB and good adherence to ETV. The results of this study showed that, without considering adherence, patients with CHB and LLV had a significantly increased incidence of HCC, in addition to other traditional risk factors in the entire cohort. On the other hand, no significant difference was found in the clinical outcomes between MVR and LLV groups among good adherence group in our study, and these results were found in poor adherence group as well (Supplementary Table 1).

To directly compare the effect of LLV and medication adherence on the development of HCC, we performed univariate and multivariate analyses (Supplementary Table 2); in addition to the traditional risk factors for HCC, such as old age, male sex, and cirrhosis, good adherence to ETV treatment was highly associated with the development of HCC with a HR of $0.40(95 \% \mathrm{Cl}, 0.25-0.66$;
$P<0.001)$. However, MVR was not independently associated with HCC $(P=0.361)$. Notably, in the case of multivariate analyses not including adherence (Supplementary Table 3), MVR was significantly associated with the development of HCC with a HR of 0.61 (95\% Cl, 0.39-0.96; $P=0.031$ ), in addition to the other risk factors: old age, male sex, and cirrhosis. On the other hand, among patients with MVR ( $n=654)$, HCC developed in 36/509 patients (7.1\%) in good adherence group and 22/145 (15.2\%) in poor adherence group during the follow-up period of up to 132 months. Kaplan-Meier survival analysis demonstrated a significantly higher risk of HCC in poor adherence group compared to good adherence group (log-rank $P<0.001)$. Liver-related death or transplantation occurred in $4 / 509(0.8 \%)$ in good adherence group and $10 / 145(6.9 \%)$ in poor adherence group. Hepatic decompensation occurred in 12/509 (2.4\%) in good adherence group and 15/145 $(10.3 \%)$ in poor adherence group. Kaplan-Meier survival analysis demonstrated a significantly higher risk of liver-related death or transplantation, as well as hepatic decompensation, in poor adherence group compared to good adherence group (log-rank $P<0.001)$.

In this study, adherence was shown to be a more significant factor than LLV for predicting the occurrence of cirrhotic complications. However, MVR vs. LLV and good vs. poor adherence are not necessarily different concepts, and both are indicators that reflect the intra-hepatic viral replication of HBV. In the statistics of our study, the result that LLV does not appear to be an independent risk factor for HCC occurrence does not infer that LLV is not related to HCC occurrence, but is rather a confounding factor closely related to adherence. The ideal viral suppression is a key mechanism to prevent cirrhotic complications in patients with CHB, such as HCC. In general, LLV is based on a serologic test for HBV DNA conducted at 3-to-6-month intervals, with the limitation that viral replication status in the middle of testing is unknown. In contrast, nonadherence refers to a period of inadequate medication during follow-up, suggesting the possibility of viral proliferation during the period of nonadherence. In other words, as sustained antiviral therapy is important for HBV inhibition, theoretically, adherence can be considered as a continuous variable that more accurately reflects intra-hepatic viral loads compared to LLV with periodic serologic testing.

A previous study of Korean patients with optimal VR during NA therapy showed that the cumulative HCC incidence was $8.1 \%$ at 3 years and $17.4 \%$ at 5 years with baseline liver cirrhosis, and $4.7 \%$ at 3 years and $7.2 \%$ at 5 years without baseline liver cirrhosis. ${ }^{9}$ In our study, the 5 - and 10 -year cumulative incidence rates of HCC 
in good adherence vs. poor adherence were 5.8 vs. $15.9 \%$ and 10.3 vs. $26.0 \%$, respectively, in the entire cohort (Supplementary Fig. 4). In terms of cirrhotic subcohort, the 5- and 10-year cumulative incidence rates of HCC in good adherence vs. poor adherence were 10.0 vs. $28.9 \%$ and 17.1 vs. $44.1 \%$, respectively (Supplementary Fig. 5). These results suggest that the long-term preventive effects of ETV treatment may be significantly attenuated in patients with poor adherence. This again presents the importance of continuous and regular medication in patients with CHB.

The strengths of this study were its large sample size and longterm follow-up, which together allowed for increased statistical power and greater reliability of data. We also sought to analyze from multiple perspectives to clarify the relationship between adherence, LLV, and cirrhotic complications. Nonetheless, this study had some limitations. First, the retrospective nature of certain clinical information might be biased due to incomplete data collection. The common reasons affecting patient adherence, such as lack of recognition, forgetfulness, overriding priorities, and emotional or cultural factors, may be expected to occur in patients prescribed NA therapy. However, due to the retrospective nature of this study, the causes could not be identified in approximately $30 \%$ of the patients. Second, individuals who developed LLV had different durations of LLV during the follow-up period, which may have had different degrees of impact on clinical outcomes. Furthermore, defining LLV or MVR is subject to the frequency and intervals of HBV DNA testing and may not be the same for all patients, which may result in classification bias. Third, since the number of patients with cirrhosis and LLV despite good adherence to treatment was very low ( $n=40$, only six cases of HCC), it was too statistically low-powered to draw a definite conclusion regarding the effect of LLV on clinical outcomes among the cirrhotic subgroup. Fourth, since our database did not include other NAs (lamivudine, adefovir dipivoxil, telbivudine, and TDF) besides ETV, the application of these results to other NAs should be considered with caution. Finally, although pharmacy refill records are objective measures and are routinely collected, they do not measure whether the participants actually took their medications. The rates of nonadherence may have been overestimated if the dispensed medications were not used. In addition, identifying and understanding the factors influencing nonadherence to medication is necessary to identify appropriate interventions.

In conclusion, no significant difference was found between MVR and LLV groups in terms of the incidence of liver-related death or transplantation, HCC, and hepatic decompensation in patients with good adherence. For patients with suboptimal virological response, it is helpful to check their adherence before considering to continue, switch, or add another drug. For patients with good adherence who experience LLV during ETV treatment, it may be unnecessary to adjust their antiviral agent immediately, and careful observation would be possible.

\section{Authors' contribution}

Study coordination and design, data collection, data analysis, statistical analysis, writing and revision of the manuscript, and approval of the final version: SBL, BRP, and EJP. Study coordination and design, data analysis, critical review of the manuscript, and approval of the final version: NHP and JWS. Data supply, critical review of the manuscript, and approval of the final version: JJ, JHP, SWJ, IDJ, and SJB. All authors had full access to all data in the study and take responsibility for the integrity of the data and the accuracy of the data analysis.

\section{Conflicts of Interest}

The authors have no conflicts to disclose.

\section{SUPPLEMENTARY MATERIAL}

Supplementary material is available at Clinical and Molecular Hepatology website (http://www.e-cmh.org).

\section{REFERENCES}

1. Grossi G, Viganò M, Loglio A, et al. Hepatitis B virus long-term impact of antiviral therapy nucleot(s)ide analogues (NUCs). Liver Int 2017;37 Suppl 1:45-51.

2. Zhang QQ, An X, Liu YH, Li SY, Zhong Q, Wang J, et al. Long-term nucleos(t)ide analogues therapy for adults with chronic hepatitis $B$ reduces the risk of long-term complications: a meta-analysis. Virol 2011:8:72.

3. Peng CY, Chien RN, Liaw YF. Hepatitis B virus-related decompensated liver cirrhosis: benefits of antiviral therapy. J Hepatol 2012:57:442-450.

4. Liaw YF, Sung JJ, Chow WC, Farrell G, Lee CZ, Yuen H, et al. Lamivudine for patients with chronic hepatitis $B$ and advanced liver disease. N Engl J Med 2004;351:1521-1531.

5. Chang TT, Liaw YF, Wu SS, Schiff E, Han KH, Lai CL, et al. Long-term entecavir therapy results in the reversal of fibrosis/cirrhosis and continued histological improvement in patients with chronic hepatitis B. 
Hepatology 2010;52:886-893.

6. Lee KS, Kweon YO, Um SH, Kim BH, Lim YS, Paik SW, et al. Efficacy and safety of entecavir versus lamivudine over 5 years of treatment: a randomized controlled trial in Korean patients with hepatitis B e antigen-negative chronic hepatitis B. Clin Mol Hepatol 2017;23:331339.

7. Hosaka T, Suzuki F, Kobayashi M, Seko Y, Kawamura Y, Sezaki H, et al. Long-term entecavir treatment reduces hepatocellular carcinoma incidence in patients with hepatitis B virus infection. Hepatology 2013;58:98-107.

8. Arends P, Sonneveld MJ, Zoutendijk R, Carey I, Brown A, Fasano M, et al. Entecavir treatment does not eliminate the risk of hepatocellular carcinoma in chronic hepatitis B: limited role for risk scores in Caucasians. Gut 2015:64:1289-1295.

9. Cho JY, Paik YH, Sohn W, Cho HC, Gwak GY, Choi MS, et al. Patients with chronic hepatitis $B$ treated with oral antiviral therapy retain a higher risk for HCC compared with patients with inactive stage disease. Gut 2014;63:1943-1950.

10. Lim YS, Han S, Heo NY, Shim JH, Lee HC, Suh DJ. Mortality, liver transplantation, and hepatocellular carcinoma among patients with chronic hepatitis B treated with entecavir vs lamivudine. Gastroenterology 2014;147:152-161.

11. Kim DS, Jeon MY, Lee HW, Kim BK, Park JY, Kim DY, et al. Influence of hepatic steatosis on the outcomes of patients with chronic hepatitis B treated with entecavir and tenofovir. Clin Mol Hepatol 2019;25:283-293.

12. Sinn DH, Lee J, Goo J, Kim K, Gwak GY, Paik YH, et al. Hepatocellular carcinoma risk in chronic hepatitis B virus-infected compensated cirrhosis patients with low viral load. Hepatology 2015;62:694-701.

13. Kim JH, Sinn DH, Kang W, Gwak GY, Paik YH, Choi MS, et al. Lowlevel viremia and the increased risk of hepatocellular carcinoma in patients receiving entecavir treatment. Hepatology 2017;66:335343.

14. Terrault NA, Bzowej NH, Chang KM, Hwang JP, Jonas MM, Murad $\mathrm{MH}$, et al. AASLD guidelines for treatment of chronic hepatitis $B$. Hepatology 2016;63:261-283.

15. Lee M, Keeffe EB. Study of adherence comes to the treatment of chronic hepatitis B. J Hepatol 2011;54:6-8.

16. Vrijens B, De Geest S, Hughes DA, Przemyslaw K, Demonceau J, Ruppar $T$, et al. A new taxonomy for describing and defining adherence to medications. Br J Clin Pharmacol 2012;73:691-705.

17. Pawlotsky JM, Dusheiko G, Hatzakis A, Lau D, Lau G, Liang TJ, et al. Virologic monitoring of hepatitis $B$ virus therapy in clinical trials and practice: recommendations for a standardized approach. Gastroenterology 2008;134:405-415.

18. Chang TT, Gish RG, de Man R, Gadano A, Sollano J, Chao YC, et al. A comparison of entecavir and lamivudine for HBeAg-positive chronic hepatitis B. N Engl J Med 2006;354:1001-1010.
19. Marcellin P, Heathcote EJ, Buti M, Gane E, de Man RA, Krastev Z, et al. Tenofovir disoproxil fumarate versus adefovir dipivoxil for chronic hepatitis B. N Engl J Med 2008;359:2442-2455.

20. Shin JW, Jung SW, Lee SB, Lee BU, Park BR, Park EJ, et al. Medication nonadherence increases hepatocellular carcinoma, cirrhotic complications, and mortality in chronic hepatitis B patients treated with entecavir. Am J Gastroenterol 2018;113:998-1008.

21. Hong SP, Kim NK, Hwang SG, Chung HJ, Kim S, Han JH, et al. Detection of hepatitis $B$ virus YMDD variants using mass spectrometric analysis of oligonucleotide fragments. J Hepatol 2004;40:837-844.

22. Sarin SK, Kumar M, Lau GK, Abbas Z, Chan HL, Chen CJ, et al. Asian-Pacific clinical practice guidelines on the management of hepatitis B: a 2015 update. Hepatol Int 2016;10:1-98.

23. Bruix J, Sherman M; American Association for the Study of Liver Diseases. Management of hepatocellular carcinoma: an update. Hepatology 2011;53:1020-1022.

24. Chotiyaputta W, Peterson C, Ditah FA, Goodwin D, Lok AS. Persistence and adherence to nucleos(t)ide analogue treatment for chronic hepatitis B. J Hepatol 2011;54:12-18.

25. Papatheodoridis GV, Dalekos GN, Yurdaydin C, Buti M, Goulis J, Arends $\mathrm{P}$, et al. Incidence and predictors of hepatocellular carcinoma in Caucasian chronic hepatitis B patients receiving entecavir or tenofovir. J Hepatol 2015;62:363-370.

26. Raffetti E, Fattovich G, Donato F. Incidence of hepatocellular carcinoma in untreated subjects with chronic hepatitis B: a systematic review and meta-analysis. Liver Int 2016;36:1239-1251.

27. Eun JR, Lee HJ, Kim TN, Lee KS. Risk assessment for the development of hepatocellular carcinoma: according to on-treatment viral response during long-term lamivudine therapy in hepatitis $B$ virusrelated liver disease. J Hepatol 2010;53:118-125.

28. Park YH, Kim BK, Kim JK, Kim HC, Kim DY, Park JY, et al. Long-term outcomes of chronic hepatitis B virus infection in the era of antiviral therapy in Korea. J Gastroenterol Hepatol 2014;29:1005-1011.

29. Abu-Amara M, Feld JJ. Does antiviral therapy for chronic hepatitis $B$ reduce the risk of hepatocellular carcinoma? Semin Liver Dis 2013;33:157-166.

30. Lee SB, Kim KM, An J, Lee D, Shim JH, Lim YS, et al. Clinical characteristics and potential aetiologies of non- $\mathrm{B}$ non-C hepatocellular carcinoma in hepatitis B virus endemic area. Liver Int 2016;36:13511361.

31. Saitta C, Tripodi G, Barbera A, Bertuccio A, Smedile A, Ciancio A, et al. Hepatitis $B$ virus (HBV) DNA integration in patients with occult HBV infection and hepatocellular carcinoma. Liver Int 2015;35:23112317.

32. Chotiyaputta W, Hongthanakorn C, Oberhelman K, Fontana RJ, Licari T, Lok AS. Adherence to nucleos(t)ide analogues for chronic hepatitis B in clinical practice and correlation with virological breakthroughs. J Viral Hepat 2012;19:205-212. 
Seung Bum Lee, et al. Low-level viremia and adherence to entecavir

33. Berg T, Marcellin P, Zoulim F, Moller B, Trinh H, Chan S, et al. Tenofovir is effective alone or with emtricitabine in adefovir-treated patients with chronic-hepatitis B virus infection. Gastroenterology 2010;139:1207-1217.

34. Lieveld Fl, van Vlerken LG, Siersema PD, van Erpecum KJ. Patient adherence to antiviral treatment for chronic hepatitis B and C: a systematic review. Ann Hepatol 2013;12:380-391.

35. Wong GL, Chan HL, Mak CW, Lee SK, Ip ZM, Lam AT, et al. Entecavir treatment reduces hepatic events and deaths in chronic hepatitis B patients with liver cirrhosis. Hepatology 2013;58:1537-1547. 